\title{
INDICS: An Industrial Internet Platform
}

\author{
Xudong Chai \\ CASICloud Co. Ltd. \\ Beijing, China, \\ xdchai@263.net
}

\author{
Baocun Hou \\ Beijing Simulation Center \\ Beijing, China \\ houbc2015@sina.com
}

\author{
Ping Zou \\ Hangtian Zhizao Co. Ltd. \\ Beijing, China
}

\author{
Jinle Zeng \\ Hangtian Zhizao Co. Ltd. \\ Beijing, China
}

\author{
Jiehan Zhou \\ (Member IEEE) \\ University of Oulu \\ Oulu, Finland \\ jiehan.zhou@oulu.fi
}

\begin{abstract}
The industrial Internet integrates the Internet, big data, artificial intelligence, and the real economy. We introduce China's first industrial Internet platform INDICS-one of the world's first industrial Internet platforms. We present the INDICS system architecture, and examine three successful INDICS application cases.
\end{abstract}

Keywords - Industrial Internet, INDICS, Internet, Big data

\section{INTRODUCTION}

The name "Industrial Internet" was first coined by General Electric (GE) as a term integrating big data, analytical tools, and wireless networks with physical and industrial equipment [1]. Recently, the Industrial Internet is embracing ideas of intelligent objects with embedded technology and the Internet of Things (IoT) $[2,3]$. This paper uses both terms interchangeably. In manufacturing, a typical Industrial Internet example is manufacturing tools equipped with IoT technologies that "talk each other" by sending data back and forth.

Industrial Internet platforms integrate information and communications technology to improve industrial efficiency. It is becoming the key to building and upgrading digital factories. The Industrial Internet platform also expects to accelerate both the migration of industrial information systems to the Cloud and the use of industrial big data analytics and applications. Industrial data intelligence is becoming a key factor in improving manufacturing productivity and competitive innovation through artificial intelligence-driven product design, production process, asset management, and service management, etc. Top Big Data Processing Frameworks include MapReduce, Hadoop, Spark, Flink, Storm, Samza, and Microsoft DryadLINQ [4-6]. There are many cloud computing platforms such as Amazon AWS, Microsoft Azure, Watson Machine learning, which offer machine learning and deep learning through cloud services for developers and data scientists to accelerate transfer and intelligent use of data [7-13].

Outside China, GE has launched Predix Industrial Internet Cloud Platform [14] and Siemens has developed MindSphere Industrial Internet Platform [15-16]. China Aerospace Science and Industry Corporation developed China's first Industrial Internet platform, INDICS [17-19], which is an industrial Internet platform for the manufacturing cloud to facilitate innovation of manufacturing technologies and to build a new industrial Internet ecosystem with a Data as a Service layer.

The remainder of the paper is organized as follows. Section 2 revisits the concepts of Industrial Internet and Industrial Internet platform. Section 3 introduces the INDICS platform. Section 4 examines three INDICS application cases. Section 5 concludes the paper and gives suggestions for directing the INDICS development.

\section{REVISITING INDUSTRIAL INTERNET}

\section{A. Industrial Internet}

The Chinese Alliance of Industrial Internet presents its Industrial Internet as a new paradigm [20]: an advanced digital networked cloud manufacturing, carrying the latest information and communication technology for intelligent decision-making and control, which improves the efficiency of manufacturing resource allocation by connecting machines, materials, people, and information systems.

The Industrial Internet should have the following three key elements [21]:

- Intelligent machines connect facilities, networks, and people around the world with sensors, controls, and software applications.

- Advanced analytics leverages professional knowledge, predictive algorithms, automation, materials science, electrical engineering, and other disciplines to understand how machines and large systems operate.

- People at work. Whether working in industrial facilities, offices, hospitals, or on the move, people should always be in touch via smarter design, so they can deliver higher quality service safely.

\section{B. Industrial Internet Platform}

The Chinese Alliance of Industrial Internet describes Industrial Internet platform as an integrated technical platform to implement the Industrial Internet [20]. It utilizes the latest information and communication technology to build an intelligent operating and development environment for industry with various digital tools such as ubiquitous networking, big data storage and processing, resource allocation and optimization, etc. In addition, the Industrial Internet platform forms a new manufacturing ecosystem with resource enrichment, multi-participation, cooperation, etc.

\section{Requirements for an Industrial Internet Platform}

With the development of manufacturing, the digital economy, information communication technology such as cloud 
computing, the Internet of Things, and Big Data, every Industrial Internet platform faces the following challenges.

- First, industrial data is growing explosively. The Industrial Internet must be able to cope with this growth, which requires new data management tools, including new tools for intelligent decision-making.

- The Industrial Internet model must encourage industrial innovation and profound business collaboration.

- New information communication technology brings a chance to revolutionize digital manufacturing from the ground up. For example, the Internet of Things helps companies efficiently manage thousands of different types of equipment. New generation artificial intelligence will enhance companies' data insight capabilities.

- The Internet is changing business models. Industry requires a platform, which can realize rapid integration and utilization of resources, quickly respond to market demands at low cost, and promote personalized customization and networked collaboration, etc.

\section{Technical characteristics of Industrial Internet platform}

The Industrial Internet platform aims at meeting the abovementioned challenges by leveraging emerging technologies such as Internet of Things, Big Data, artificial intelligence, 5G, etc.. Based on the traditional cloud platform with the basic technical features of IaaS (Infrastructure as a Service), PaaS (Platform as a Service) and SaaS (Software as a Service), the industrial Internet platform also has the following four characteristics:

- Ubiquitous connectivity: the ability to collect various production elements such as equipment, software, and personnel.

- Data as a Service: enables massive data storage, management, and processing based on cloud computing architecture.

- Knowledge accumulation: provides data analysis capabilities based on industrial domain knowledge, and support knowledge solidification, accumulation and reuse.

- Application innovation: allows the user to access platform functions and resources, to provide the user with an open environment for industrial APP development and innovation.

\section{INDICS ARCHITECTURE}

This section introduces INDICS architecture based on the theoretic analysis in Section 2.

\section{A. Background}

In June 2015, China Aerospace Science and Industry Corporation established CASICloud Co., Ltd [22]. In June 2017, their Industry Intelligent Cloud System (INDICS), an industrial Internet platform was officially released, and INDICS became China's first Industrial Internet platform.

Currently, INDICS is the Industrial Internet platform with the largest number of registered enterprises and the largest number of connected devices in the world. The number of registered companies exceeds 1.4 million, covering 366 industries such as the communication equipment industry, electrical machinery, general equipment, textiles, special equipment, metal-works, and the information technology industry. With the INDICS platform, nearly 170 billion RMB has been proposed for the cooperation and nearly 80 billion RMB succeeded.

\section{B. INDICS architecture}

The INDICS infrastructure consists of five layers: the resource layer, industrial Internet of Things layer, access layer, cloud layer, and the industrial APP layer (see Fig. 1).

Resource Layer. This layer supports the connection to industrial services, industrial equipment, and industrial products. The connected industrial services include 14 categories of manufacturing capacity with 66 sub-categories, 12 categories of testing capability with 139 sub-categories, and 3 categories of metrology with 30 sub-categories. The connected industrial equipment includes 21 categories, such as mechanical processing, environmental testing, and simulation tests.

Industrial IoT Layer. This layer provides industrial equipment with communication interconnection, which supports the mainstream industrial communication protocols such as OPC-UA [23], MQTT [24], Modbus [25], and Profinet [26], and supports the communication of industrial field buses, wired networks, and wireless networks as well.

Access Layer. This layer provides Smart IOT series intelligent gateways and INDICS-API with our own intellectual property rights, which support data processing combining cloud computing and edge computing. Through the open standard interface, the INDICS platform connects more than 25,000 devices, including more than 10,000 manufacturing equipment, and enable to collect various types of data such as equipment identification, operation status, and events of various industrial resources.

Cloud Layer. This layer provides IaaS for cloud infrastructure management, DaaS for big data storage/management/analysis, and general PaaS and industrial PaaS services.

a) The IaaS sub-layer has a data center with the data security level 3, 1,217 physical servers, 132 storage devices, and 363 network devices, 96,000 VCPUs and 9.21PB storage capacity.

b) The DaaS layer provides big data storage capabilities in different formats supported by Hadoop [27], HBase [28], and Cassandra [29] as well as big data analysis capabilities such as Apache Storm [30] and Apache Spark [31], which helps industrial big data analysis and business intelligence.

c) The PaaS layer uses the mainstream open source Cloud Foundry [32] as the underlying infrastructure, providing application development tools such as HyperTransport Extension 3 (HTX3) [33] and integration development environment, messaging mechanisms, caching, search, AI algorithms, and big data algorithm library, etc. The PaaS layer has more than 200 APIs supporting application lifecycle management, and provides industrial service and workflow engines, data analysis and simulation engines for software-defined manufacturing. At the same time, the PaaS layer enables third-party platforms to access the INDICS platform. 


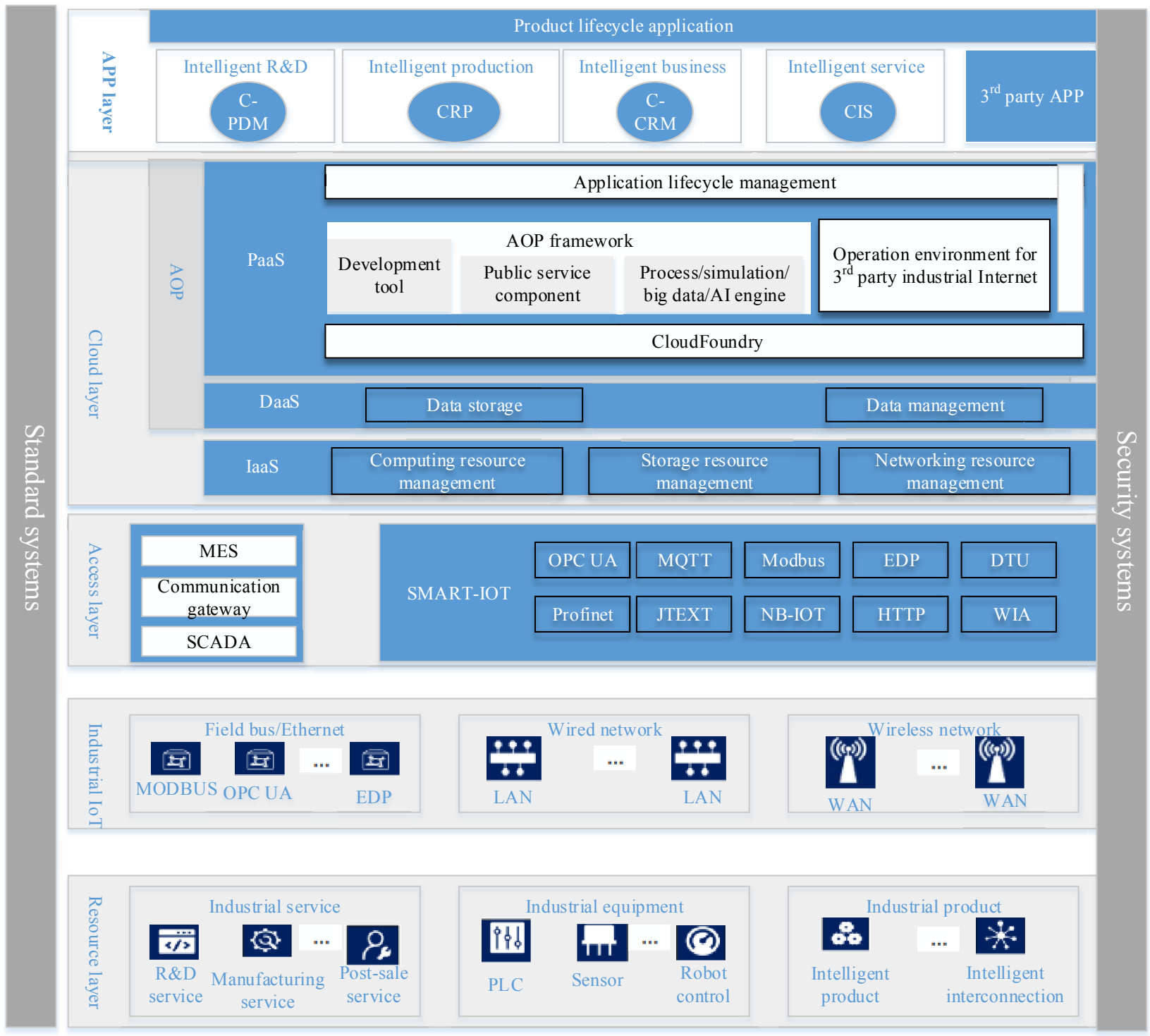

Figure 1. INDICS platform architecture

APP Layer. This layer provides industrial application services, including intelligent business, intelligent $R \& D$, intelligent management and control, and intelligent industrial services such as remote monitoring, intelligent diagnosis, postsales service, and asset management. The INDICS platform has more than 500 kinds of software and applications open to the users (ranked first in the world), covering the entire industrial chain of product $R \& D$, lean manufacturing, intelligent services, smart companies, etc.

\section{INDICS APPLICATION CASES}

The INDICS platform provides small to medium enterprises (SME) with successful access to intelligent factories, the industrial cloud, industrial big data, and intelligent services. This section examines three INDICS-based application cases.

\section{A. Intelligent factory.}

An intelligent factory can help SMEs to upgrade their digital and intelligent manufacturing systems. We have coordinated an intelligent factory into various SMEs ranging from aerospace, electronics, chemical, medical, automotive, etc. We have provided 21 professional solutions for machining, electrical equipment, testing, environmental testing, and achieved good results. 
For example, in the case of Henan Aerospace Hydraulic \& Pneumatic Technology Co., Ltd. [34], with the services of CPDM, CRP and virtual factories provided by the INDICS platform, the company upgraded and built an automated flexible production line consisting of 4 sets of $12 \mathrm{CNC}$ machining centers for loading and unloading, pre-assembly, pre-adjustment, and AGV-aided material. This solution achieved the goals of online business matching, collaborative design, intelligent scheduling, offline intelligent manufacturing, and online process control and monitoring.

\section{B. Industrial cloud}

In response to uneven levels of regional informatization, and to weak industrial value chain and business collaboration, industrial cloud solutions must adapt to differences in regional economic development. At present, the INDICS platform has established industrial cloud platforms that meet the requirements ranging from furniture manufacturing, mould, and other industries, enhancing their resource allocation efficiency. We simultaneously built industrial cloud platforms in Guizhou, Anhui, Inner Mongolia, and Jiangxi - areas with wide differences in technological development and cultures.

For example, in the implementation of Anhui Industrial Cloud, we selected capacity, demand, cloud-based design, and software sharing as its main services. Since its launch, it has drawn more than 3,985 enterprises from Anhui government affairs to Anhui Industries. The platform gets 169 views per day.

In the implementation of Guizhou Industrial Cloud (Figure 2) [35], in addition to the services provided by the Anhui Industrial Cloud, we added integrated services, industrial services, and cloud-based manufacturing. Since its launch, more than 70,000 enterprises have registered. There are more than 15,000 pieces of information on the supply and demand for industrial products, more than 25,000 products, and a cumulative amount of nearly 2 billion RMB, with a cumulative turnover of 1 billion RMB. The Guizhou Industrial Cloud is listed as the first pilot integrating manufacturing and Internet by the Chinese Ministry of Industry and Information Technology.

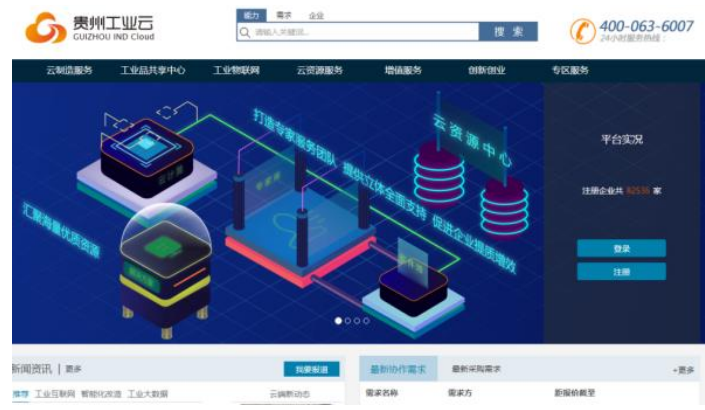

Figure 2. Guizhou Industrial Cloud platform

\section{Industrial Big Data and Intelligent Service}

In order to guarantee product quality throughout the product life cycle, INDICS provides companies with big data analysis for equipment performance monitoring (Fig.3).
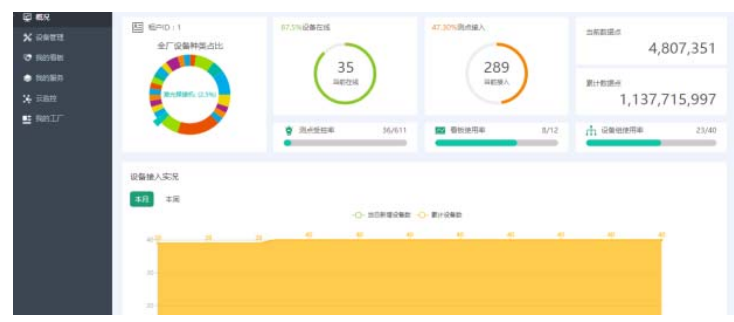

Figure 3. Equipment performance monitoring

For example, in the solution to monitoring robots for a $\mathrm{CNC}$ company, INDICS connects 266 robot devices for monitoring their operation status. This effectively reduces unplanned shutdowns, and optimizes robot operation through fault data analysis. In another solution to a real-time data monitoring for grid fans, INDICS connects 1022 fans, uploads 200 million pieces of data each day. This provides equipment failure warning, and power evaluation for a smart wind power farm.

\section{CONCLUSION AND FUTURE}

The Industrial Internet integrates Big Data, analytical tools, and industrial equipment. The Industrial Internet is the core for achieving Industry 4.0 and Made in China 2025. At present, only GE and Siemens have developed their Industrial Internet outside of China. INDICS is China's first industrial Internet platform. In this paper, we revisited the definition of Industrial Internet and Industrial Internet platform. Then we presented INDICS platform architecture and examined its use in the intelligent factory, industrial cloud, and industrial Big Data. Those cases prove that INDICS improves production effectiveness and efficiencies by integrating intelligent manufacturing equipment, reducing costs, and guaranteeing product quality with the use of data analysis and forecasting.

In the future, we will improve INDICS from the following directions:

1) Improve INDICS platform services for the five core industrial engines: industrial service engine, process-oriented engine, big data analysis engine, simulation, and AI engine.

2) Strengthen the openness of INDICS platform. On the one hand, we will improve the user interfaces in the management of data, models, and services for facilitating APPs development. On the other hand, we improve the support and interconnection for different protocols such as OPC-UA, MQTT, Modbus, and Profinet.

3) R\&D on blockchain-based INDICS security systems guaranteeing secure access to device, platform, application, data, and network.

4) R\&D on edge computing-based high-performance intelligent gateways, and introducing an intelligent gateway product, which supports edge computing. 


\section{REFERENCES}

[1] Industrial Internet. https://www.techopedia.com/definition/30044/industrial-internet, accessed by 18.03.2018.

[2] S.Jeschke, C.Brecher, T.Meisen, D. Özdemir, T. Eschert. Industrial internet of things and cyber manufacturing systems. In Industrial Internet of Things, pp. 3-19, 2017.

[3] J.Zhou, T. Leppanen, E. Harjula. M.Ylianttila, T.Ojala, C. Yu, L. Yang. Cloudthings: A common architecture for integrating the internet of things with cloud computing. In IEEE Computer Supported Cooperative Work in Design (CSCWD), pp. 651-657, 2013.

[4] P. Chandarana, M. Vijayalakshmi.. Big data analytics frameworks. In IEEE 2014 international conference on Circuits, Systems, Communication and Information Technology Applications (CSCITA), pp. 430-434.

[5] W. Zhang, Y. Zhang, J. Zhai, D. Zhao, L. Xu, J. Zhou, Z. Li. Multi-source data fusion using deep learning for smart refrigerators. Computers in Industry, vol. 95, pp.15-21,2018.

[6] W. Zhang, W. Guo, X. Liu, Y. Liu, J. Zhou, B. Li, Q. Lu. LSTM-Based Analysis of Industrial IoT Equipment. IEEE Access, vol. 6, pp. 2355123560.2018 .

[7] IBM. Machine Learning Services. http://www.ibm.com/Watson, accessed by 18.03 .2018 .

[8] S. Mund. Microsoft azure machine learning. Packt Publishing Ltd, 2015.

[9] Amazon. Machine Learning on AWS. https://aws.amazon.com/machinelearning/, accessed by 18.03.2018.

[10] J. Peng, X. Zhang, Z. Lei, B. Zhang, W. Zhang, Q. Li, Comparison of several cloud computing platforms. In IEEE Second International Symposium on Information Science and Engineering (ISISE 2009), pp. 2327, 2009.

[11] W. Zhang, G. Wulan, J. Zhai, L. Xu, D. Zhao, X. Liu, S. Yang, J. Zhou. An intelligent power distribution service architecture using cloud computing and deep learning techniques. Journal of Network and Computer Applications, vol. 103, pp. 239-248, 2018.

[12] W. Zhang, H. Lv, L. Xu, X. Liu, J. Zhou. Data Mining as a Cloud Service for Learning Artificial Intelligence. In Pacific-Rim Symposium on Image and Video Technology, pp. 214-221, Springer, 2017.

[13] W. Zhang, L. Chen, Q. Lu, Y. Rao, J. Zhou. Towards an osgi based pervasive cloud infrastructure. In IEEE Green Computing and Communications (GreenCom), pp. 418-425, 2013.

[14] N. Agarwal, A. Brem. Strategic business transformation through technology convergence: implications from General Electric's industrial internet initiative. International Journal of Technology Management, 67(24), pp. 196-214, 2015.

[15] R. Nelson. Smart factories leverage cloud, edge computing. EE-Evaluation Engineering, 56(6), pp. 14-18, 2017.

[16] N.A. Dumitrascu, D. Alexandru. Digitial Transofrmation of Industrial Companies in the Context of Inudstry 4.0. In Conference Proceedings of the Academy of Romanian Scientists. Vol. 9, No. 1, pp. 9-18, 2017.

[17] CASICloud. INDICS. http://intl.indics.com/, accessed by 18.03.2018.

[18] B. Li, L. Zhang, L. Ren, X. Chai, F. Tao, Y. Luo, X. Zhao. Further discussion on cloud manufacturing. Computer integrated manufacturing systems, 17(3), 449-457, 2011.

[19] T. Fei, Y. Cheng, L. Xu, L. Zhang, B. Li. CCIoT-CMfg: cloud computing and internet of things-based cloud manufacturing service system. IEEE Transactions on Industrial Informatics, 10(2), 1435-1442, 2014.

[20] The Chinese Alliance of Industrial Internet. Industrial Internet Version 1.0. Architecture. http://en.aii-alliance.org/, accessed by 18.03.2018.

[21] GE. Industrial Internet:Pushing the Boundaries of Minds and Machines https://www.ge.com/docs/chapters/Industrial_Internet.pdf, accessed by 18.03.2018.

[22] CASICloud. http://www.casicloud.com/, accessed by 18.03.2018.

[23] T.Hannelius, M.Salmenpera, S.Kuikka. Roadmap to adopting OPC UA. In IEEE Industrial Informatics, pp. 756-761, 2008.

[24] U. Hunkeler, H. Truong, A. Stanford-Clark. MQTT-S-A publish/subscribe protocol for Wireless Sensor Networks. In IEEE Communication systems software and middleware and workshops, pp. 791-798, 2008
[25] P.Huitsing, R.Chandia, M.Papa, S.Shenoi. Attack taxonomies for the Modbus protocols. International Journal of Critical Infrastructure Protection, 1, 37-44. 2008

[26] J. Feld. PROFINET-scalable factory communication for all applications. In IEEE Factory Communication Systems, pp. 33-38, 2004.

[27] K.Shvachko, H.Kuang, S.Radia, R. Chansler. The hadoop distributed file system. In IEEE 26th symposium on Mass storage systems and technologies (MSST), pp. 1-10, 2010.

[28] M. N. Vora. Hadoop-HBase for large-scale data. In 2011 international conference on Computer science and network technology (ICCSNT), Vol. 1, pp. 601-605, 2011.

[29] G.Wang, J.Tang. The nosql principles and basic application of cassandra model. In 2012 International Conference on Computer Science \& Service System (CSSS), pp. 1332-1335, 2012.

[30] Storm. http://storm.apache.org/, accessed by 18.03.2018.

[31] Spark. http://spark.apache.org/sql/, accessed by 18.03.2018.

[32] D. Bernstein. Cloud foundry aims to become the OpenStack of PaaS.IEEE Cloud Computing, 1(2), 57-60, 2014.

[33] HyperTransport. https://en.wikipedia.org/wiki/HyperTransport, accessed by 18.03 .2018 .

[34] Henan Aerospace Hydraulic \& Pneumatic Technology Co., Ltd. http://en.hnhtcng.com/about.aspx, accessed by 18.03.2018.

[35] Guizhou IND Cloud. http://www.gz-icloud.com.cn/, accessed by 18.03.2018. 